\title{
A Self-learning Knowledge based System for Diagnosis and Treatment of Chronic Kidney Disease
}

\author{
Siraj Mohammed \\ College of Engineering and Technology, Jigjiga University, Ethiopia
}

Received: 10 July 2018; Accepted: 17 December 2018; Published: 08 March 2019

\begin{abstract}
Chronic kidney disease is an important challenge for health systems around the world and consuming a huge proportion of health care finances. Around $85 \%$ of the world populations live in developing country of the world, where chronic kidney disease prevention programs are undeveloped. Treatment options for chronic kidney disease are not readily available for most countries in sub-Saharan Africa including Ethiopia. Many rural and urban communities in Ethiopia have extremely limited access to medical advice where medical experts are not readily available. To address such a problem, a medical knowledge-based system can play a significant role. Therefore, the aim of this research was developing a self- learning knowledge based system for diagnosis and treatment of the first three stages of kidney disease that can update the knowledge without the involvement of knowledge engineer. In the development of this system, the following procedures are followed: Knowledge Engineering research design was used to develop prototype system. Purposive sampling strategies were utilized to choose specialists. The information was acquired by using both structured and unstructured interviews and all knowledge's are represented by using production rule. The represented production rule was modeled by using decision tree modeling approach. Implementation was employed by using pro-log tools. Testing and evolution was performed through test case and user acceptance methods. Finally, we extensively evaluate the prototype system through visual interactions and test cases. The test results show that our approach is better to the current ones.
\end{abstract}

Index Terms: Knowledge-based system, Kidney diseases, machine learning, Knowledge Engineering, Knowledge Representation.

(C) 2019 Published by MECS Publisher. Selection and/or peer review under responsibility of the Research Association of Modern Education and Computer Science.

\footnotetext{
* Corresponding author.

E-mail address: sirmoh4@gmail.com
} 


\section{Introduction}

Chronic kidney disease is an important challenge for health systems around the world and consuming a huge proportion of health care finances. It is even more significant for developing countries which now face the double burden of infectious diseases and growing problems of non- communicable diseases such as cardiovascular, diabetes and hypertension [1]. Around $85 \%$ of the world populations live in developing country of the world [2]. Treatment options for chronic kidney disease are not readily available for most countries in sub-Saharan Africa including Ethiopia. For many years the treatment and diagnoses chronic kidney disease in Ethiopia has not been studied and there is no national strategy for prevention and care of patients with chronic kidney disease. Among the people of developing countries like Ethiopia, chronic kidney disease are the one the increase causes of death, because in Ethiopia there is no well-established chronic kidney disease prevention programs and a shortage of specialists and health facilities are there. Therefore, it is difficult to diagnosis and treatment of chronic kidney disease in such like conditions.

Artificial intelligence has emerged after the introduction of the first computers. Currently, the concept of the artificial intelligence is understood as a branch of computer science, in which the computer programs have capability to simulate human behaviors. Computer programs are used both for experimental and practical purposes, such as formulation of medical diagnoses. KBS is one of braches of the AI groups. A knowledgebased system is a software system that contains a significant amount of knowledge in an explicit, declarative form. This has now been replaced by methodological approaches that have many similarities with general software engineering practice. KBS development is best seen as software engineering for a particular class of application problems. These applications problems typically require some form of reasoning to produce the required results. In current business practice there is an increasing need for such systems, due to progression of information technology in our daily work. Some typical applications are systems for diagnosing problems in medical sections [3]. Knowledge based system has become as platform to solve real problem in a fashion similar to the expert in medical areas. Knowledge based system is a computer program which captures the knowledge of experts on a given problem to solve problems in a fashion similar to the expert.

The system can assist the expert during problem-solving, or act like expert in situations where the expertise is lacking. The commonly used knowledge acquisition techniques are interviews. Interviews can be classified into structure, semi-structure and unstructured, observation and document analysis. Learning technologies to develop KBS are neural networks, intelligent agents, fuzzy logic, and genetic learning and self-learning [4]. Just a few research papers are accessible that have the capacity of self-learning with KBS. In broad aspect, the self-learning field is called as machine learning in which the computer programs learn from their own experience upon utilization. Self-learning is one of the elements of KBS which tries to imitate the learning capability of human beings. It is possible to update the knowledge base of the KBS either manually or automatically using machine learning algorithms [5, 6]. According to Akerkar, Sajja and Solomon G. [5, 7], "Self-learning is a scientific task that enables the knowledge-based system to learn automatically from the inference process". The aim of this research was to develop a self-learning knowledge-based system to help the patients and physicians for diagnosis and treatment for kidney diseases. The prototype system is provided to the end users via computers. The key contributions of this paper are as follows.

$\checkmark$ Acquired Real facts data form physicians and modeling all facts to integration with knowledge rules using a self-learning approach.

$\checkmark$ Knowledge analysis: the process of making sense of the knowledge was performed.

$\checkmark$ Knowledge structuring: The processes of expressing the analyzed knowledge in an understandable and usable form are presented.

$\checkmark$ Knowledge modeling: the process of connecting the decision flow of the acquired knowledge and relationship between concepts was presented.

$\checkmark$ Expressing knowledge in a format of rule representation techniques. 
$\checkmark$ Design and development of a flexible self-learning knowledge-based system.

The rest of this paper is organized as follows. In Section 2, we provide an overview of the Statement of the Problem. Sections 3 describe the objective of this paper. In section 5 describe the review of related works. In Section 6, we describe the methodology of each of the key components of the prototype system in detail and the implementation of the prototype system. Section 6 considers a case study and user visual interaction approaches to evaluate the proposed system. The evaluation results are analyzed and presented for better understanding. Section 7, 8 concludes the work with implications and possible future research directions.

\section{Statement of the Problem}

Chronic kidney disease is an important challenge for health systems around the world and, consuming a huge proportion of health care finances. It is even more significant for developing countries which now face the double burden of infectious diseases and growing problems of non- communicable diseases such as cardiovascular, diabetes and hypertension [8]. Treatment options for chronic kidney disease are not readily available for most countries in sub-Saharan Africa including Ethiopia. For many years the treatment and diagnoses chronic kidney disease in Ethiopia has not been studied and there is no national strategy for prevention and care of patients with chronic kidney disease. Among the people of developing countries like Ethiopia, chronic kidney disease is the one the increase causes of death, because in Ethiopia there is no wellestablished chronic kidney disease prevention program. Unfortunately, the nature of chronic kidney disease is can progress to end stage, which is renal disease. In our country, there are no sufficient numbers of specialists and medical doctors [9]. Thus, all patients do not get enough diagnoses and treatment on time. In addition, a lot of people in Ethiopia were only known that they had this kidney disease when their kidney become chronic and affected their life. They think that all symptoms that they have were just normal fever and can be fine when they take some pills. That is a bad attitude and there are some factors that encourage to this bad attitude such as the traveling distance to the clinic or hospital, takes a lot of time in waiting especially in governmental hospital. Usually, most of the medical data Collected from patients are just saved in files or kept in folders, but those huge amounts of messy medical records have not meaning for users. Using learning KBS technique will solves the existing problem by analyzing and reusing a previous knowledge to turn that knowledge into useful knowledge base system for the diagnosis of kidney disease. Therefore, this system could help physicians, health professionals and patient to determine the diagnosis and treatment of the patient using their own language, which is Amharic language. The significance of conducting this study is to support physicians and patients in the diagnosis and treatment of kidney patients, particularly in our county where more than $85 \%$ of the Ethiopian population lives in rural areas [10]. Therefore, as stressed in the problem statement, the general issue that needs to be addressed is the possibility of integrating "a self- learning approach" with "rule-based approach" to develop a self-learning KBS for diagnosis and treatment of CKD. At the end, this study will answer the following research questions:

$>$ What type of knowledge is required to design a self-learning knowledge-based system?

$>$ What are the suitable approaches to acquired knowledge from experts?

$>$ What are the suitable models, representation techniques and implementing tools for a self- learning Amharic based knowledge-based system?

$>$ How to model and represent the acquired knowledge to developing a self- learning Amharic based knowledge-based system?

$>$ How to design and implement learning knowledge-based system techniques that are automatically updates its knowledge from experience?

$>$ How to measure the performance of the prototype system. 


\section{Objective}

\section{A. General Objectives}

The general objective of this study is to design and develop a self-learning Amharic based knowledge based system that can provide advice through Amharic user interface for physicians and patients in order to facilitate the diagnosis and treatment of kidney disease.

\section{B. Specific objectives}

To achieve the general objective of this study, the general objective should be described as the following sub specific tasks:

$\checkmark$ To review literatures on the concept of knowledge based system.

$\checkmark$ To acquire knowledge in this area.

$\checkmark$ To identify a set of learning processes.

$\checkmark$ To model and represent acquired knowledge.

$\checkmark$ To develop learning knowledge-based system for diagnosis and treatment of kidney diseases.

$\checkmark$ To evaluate and validate the performance of the prototype system.

$\checkmark$ To draw conclusions based on the findings and forward appropriate recommendations as future research directions.

\section{Significance}

This research is significant in several sides. In user side, the beneficiaries of the system are physicians and patients. The prototype system is very important as a training tool in the areas where shortages of skilled experts are available. It is also advantageous for a rural area that has the scarcity of medical professionals and medication facilities. In system developer side, the result of this study will be used as an input for the development of a full knowledge based system and it could be one approach for knowledge acquisition techniques to develop case-based reasoning applications.

\section{Review of Related Works}

Chronic kidney disease (CKD) is a condition in which the kidneys are damaged and cannot filter blood as well as possible. This damage can cause wastes to build up in the body and lead to other health problems, including cardiovascular disease (CVD), anemia, and bone disease. People with early CKD tend not to feel any symptoms [11]. The only ways to detect CKD are through a blood test to estimate kidney function, and a urine test to assess kidney damage. CKD is usually an irreversible and progressive disease and can lead to kidney failure, also called End Stage Renal Disease (ESRD), over time if it is not treated The stages of chronic kidney disease are determined by the glomerular filtration rate. Glomerular filtration is the process by which the kidneys filter the blood, removing excess wastes and fluids. Glomerular filtration rate (GFR) is a calculation that determines how well the blood is filtered by the kidneys. It is one way to measure kidney function. Glomerular filtration rate is usually calculated using a formula that includes a person's age, gender, race, and serum creatinine levels. A knowledge-based system (KBS) is a software system that contains a significant amount of knowledge in an explicit, declarative form. The area of KBS development has matured over the past two decades. It started with first-generation expert systems with a single flat knowledge base and a general reasoning engine, typically built in a rapid-prototyping fashion. This has now been replaced by methodological approaches that have many similarities with general software engineering practice. KBS development is best 
seen as software engineering for a particular class of application problems. These applications problems typically require some form of reasoning to produce the required results. In current business practice there is an increasing need for such systems, due to progression of information technology in our daily work. Some typical applications are systems for assessing loans in a bank, for job-shop scheduling in a factory, for configuring an elevator, and for diagnosing problems in a production line [12].

Moreover, the related works conducted by local and global researchers in the medical domain have been reviewed, such as E.K.juuso and k.Leiviskä [13] proposed an "Adaptive Expert Systems for Metallurgical Processes" to integrate rule-based system with fuzzy models for adaptive system, prototype KBS in antiretroviral therapy [14], designing a KBS for blood transfusion [15], prototype KBS for anxiety mental disorder diagnosis [16], the potential for applying KBS for diagnosis of acute respiratory tract infections [17], human disease diagnosis using a fuzzy expert system [18] and development of online children skin diseases diagnosis system [19] in Ethiopia. Furthermore, to the knowledge of the researchers there is no a self-learning KBS for diagnosis and treatment of CKD diseases. Therefore, the objective of the study is to develop a selflearning KBS for diagnosis and treatment of CKD.

\section{A. Learning Technologies}

Learning system may incorporate neural networks, fuzzy logic, genetic learning, self-learning KBS, or a combination of any or all of these technologies. Four state-of-the-art learning technologies are discussed as follows. These are neural networks, fuzzy logic, genetic learning, and self-learning knowledge-based system.

\section{Methodology}

The details of the research methodology appeared in the following sections, but an outline is being provided in this section. Methodological steps followed for this research are:

$\checkmark \quad$ Formulation of the problem and its justifications.

$\checkmark$ Knowledge was acquired: Using structural, unstructured interviews with experts and from existing document.

$\checkmark$ Develop a conceptual design for concept formation using decision tree and productive rule for rule representations techniques.

$\checkmark \quad$ Implement a self-learning prototype system: Architecture for prototype system and implementation part of the system was developed based on the conceptual design.

$\checkmark \quad$ Testing and evaluations of the prototype system through visual interactions and test case were carried out.

$\checkmark \quad$ Refinement: Refine the prototype system based on the outcomes of the testing and evaluation result and repeat all steps if necessary.

\section{A. Study Area, Population and Sampling Technique}

The study area for this research is Gondar University specialization hospital. Gondar University hospital is located in Amhara regional state from Ethiopia, in Gondar town at Gondar University medical campus. The size population is six. We used purposive sampling technique to select domain experts for knowledge acquisitions. The reasons to select six domain experts are to share their expertise and experience.

\section{B. Source of Data}

We used primary and secondary data as source of data. The primary data was collected by using interviews with domain experts and the secondary data was collected from published, public or private documents, books, 
journals articles, different past researches, reports, manuals and online materials.

\section{Data Collection Methods and Implantation Tool}

In this study, the necessary knowledge was acquired and elicited using interviews with the medical experts, particularly the physicians in the area of kidney disease in which six domain experts were selected from Gondar hospital based their professions, educational qualification level, and years of experience on kidney diseases. The interview with experts covered issues like, how the expert interacts with patient, what are the basic symptoms are, what techniques used to identify the patient stages, the procedures of diagnosing and what are the possible treatments recommended to the patient. A secondary data from documents such as medical books, training manuals, public or private documents, reports, online materials and journal medical articles was also assessed. Furthermore, demonstration and direct observation were considered to acquire the necessary knowledge.

Prolog language is chosen as implementation tool because it is suitable for rule based programming, backward chaining execution and pattern matching.

\section{Model}

Knowledge is to be modeled at a conceptual level. Typically, a knowledge model provides formats for writing down both static domain knowledge (rules, classes, relations) and reasoning strategies [20]. According to Richard et al. [21], one of the most extensively applied methods for conceptual modeling is decision tree. Decision tree commonly acts a key role in a knowledge modeling process. Fig 1 shows the decision tree structure that has the flow of knowledge in the diagnosis and treatment of CKD.

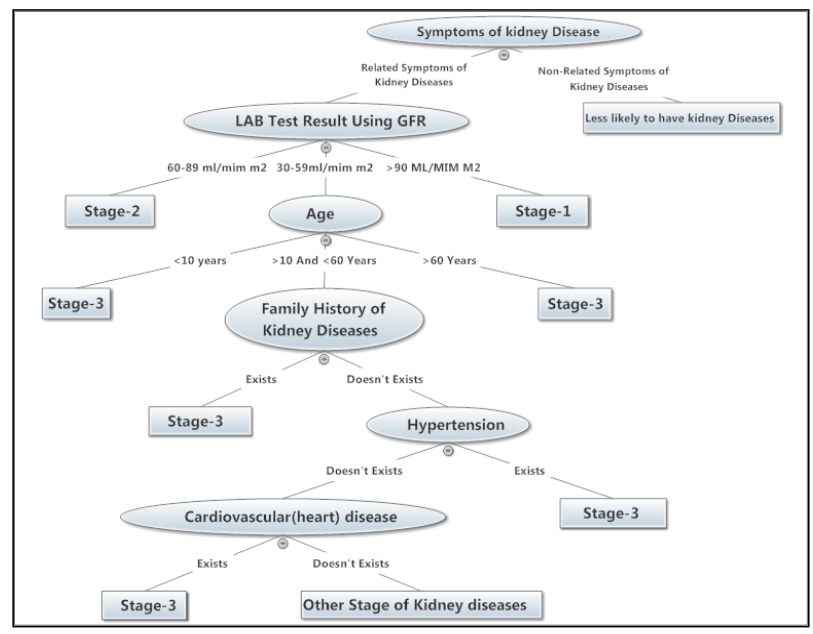

Fig.1. Decision Trees for Diagnosis and Treatment of Kidney Diseases.

\section{E. Knowledge Representation}

In the Previous section knowledge has been acquired and modeled; the next step is knowledge representation by using appropriate format that is understandable by inference engine. Knowledge representation is a means of encoding the human expert knowledge in an appropriate way. We used rule-based knowledge representation techniques to represent the acquired knowledge. All rules that are used in this study are developed based on the concepts of knowledge modeling approaches. To setup all rules, production rule Knowledge representation 
techniques are used in which knowledge is represented in the form of condition-action pairs: In the same way, the rules that contain the stages of kidney diseases, major symptoms of kidney diseases, GFR laboratory values, family history of kidney, diabetes, and cardiovascular, and high blood pressure diseases are formulated in the following ways:

\section{Rule 1:}

IF THEN

Symptoms of kidney disease = "Non-related symptoms of kidney disease",

Kidney Stage = "Kidney Stage Disease Free".

Rule 2:

IF

Symptoms of kidney disease = "Related symptoms of kidney disease"

AND Lab test result using GFR $=$ " $\geq 90 \mathrm{~mL} / \mathrm{min} / 1.73 \mathrm{~m}^{2}$ ",

THEN

Kidney Stage = "Kidney Stage 1".

Rule 3:

IF

Symptoms of kidney disease = "Related symptoms of kidney disease"

AND Lab test result using GFR = "60-89 $\mathbf{m L} / \mathbf{m i n} / \mathbf{1 . 7 3 ~} \mathrm{m}^{2} "$,

THEN

Kidney Stage = "Kidney Stage 2"

Rule 4:

IF

Symptoms of kidney disease = "Related symptoms of kidney disease"

AND Lab test result using $G F R=30-59 \mathrm{~mL} / \mathbf{m i n} / 1.73 \mathrm{~m}^{2}$,,

THEN

Kidney Stage = "Kidney Stage 3"

Rule 5:

IF

Symptoms of kidney disease $=$ "Related symptoms of kidney disease"

AND Lab test result using GFR $=$ "30-59 $\mathrm{mL} / \mathrm{min} / 1.73 \mathrm{~m}$ ",

AND Age $=$ " $\geq 60$ years",

THEN

Kidney Stage = "Kidney Stage 3"

Rule 6:

IF

Symptoms of kidney disease $=$ "Related symptoms of kidney disease"

AND Lab test result using GFR $=$ "30-59 mL/min $/ 1.73 \mathrm{~m}^{2}$ "

AND Age $=">10$ and $<=60$ years"

AND Family history of kidney disease = "Exists", 
THEN

Kidney Stage = "Kidney Stage 3"

Rule 7:

IF

Symptoms of kidney diseases = "Related symptoms of kidney diseases"

AND Lab test result using GFR = "30-59 $\mathbf{~ L L} / \mathbf{m i n} / \mathbf{1 . 7 3 ~ m}$ ",

AND Age $=$ " $\geq 10$ and $<=60$ years",

AND Family history of kidney disease = "Doesn't exist",

THEN

AND Hypertension = "Exists",

Kidney Stage = "Kidney Stage 3"

Rule 8:

IF

Symptoms of kidney disease $=$ "related symptoms of kidney disease"

AND Lab test result using GFR = "30-59 $\mathbf{m L} / \mathbf{m i n} / 1.73 \mathbf{~ m}^{2}$,"

AND Age $=" \geq 10$ and $<=60$ years",

AND Family history of kidney disease = "Doesn't exist"

AND Hypertension = "Doesn't exist"

AND Cardiovascular (heart) diseases = "Exists",

THEN

Kidney Stage = "Kidney Stage 3"

Rule 9:

IF

Symptoms of kidney disease = "Related symptoms of kidney disease"

AND Lab test result using GFR = "30-59 $\mathbf{m L} / \mathbf{m i n} / \mathbf{1 . 7 3 ~} \mathbf{~ m}^{2}$ ",

AND Age $=">10$ AND $\leq 60$ years"

AND Family history of kidney disease = "Doesn't exist"

AND Hypertension = "Doesn't exist"

AND Cardiovascular (heart) diseases = "Doesn't exist",

THEN

Kidney Stage = "other Kidney Stage"

\section{F. Implementation and Experimentations}

In this section we presented the implementation of the prototype system. Knowledge was acquired using both structured and unstructured interviews with domain experts and a relevant document was assessed by using documents analysis method to get concepts and facts about kidney diseases. Then, those extracted facts and concepts were modeled by using decision tree approach and the model has been converted into productive rules and coded by using SWI-Prolog tool to form the self-learning knowledge-based system.

\section{G. Architecture of the Prototype System}

System architecture is a conceptual model that defines the structure and guidelines of the system. The overall architecture of our system is present in Fig 2. 


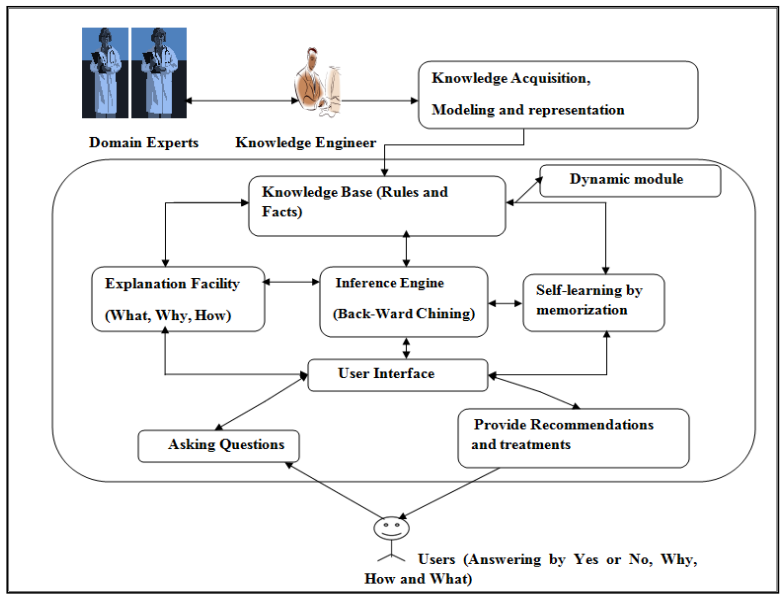

Fig.2. Architecture for Prototype System.

Figure 2, represents the structure of the prototype knowledge-based system with its main components. The architecture of such a self-learning knowledge-based system consists of some major components that are common for all knowledge-based system such us: human expert, knowledge-base, knowledge engineer, users, but in our system, two important component are added to enhance the knowledge based system such as, Selflearning with dynamic module and explanation facility components.

\section{H. Self-Learning Components of the System}

The prototype system has a capability of adding new facts/rules, signs and symptoms, patient history at run time and automatically update the old facts by replacing the new one without knowledge engineer involvements. Subsequently, self-learning module provides dynamic knowledge acquisition techniques to hold fact like, Stages of kidney disease, new symptoms, and the history of patient (name, age, address). Figure 3, show how to learn patient history and remember all facts after diagnosis and treatments.

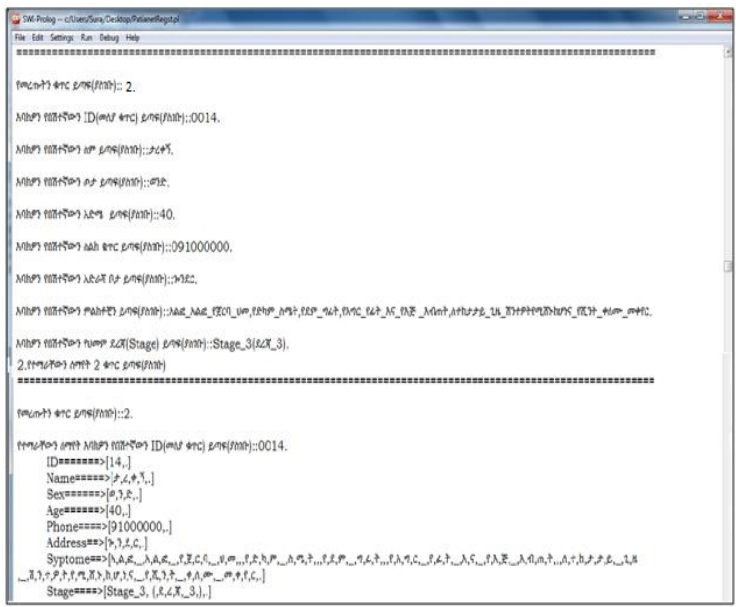

Fig.3. Illustrates How to Learn Patient History and Remember All Facts After Diagnosis and Treatments. 
The above sample window dialogue has ability to learn new sign, symptoms, and patient history and remember all facts after diagnosis and treatments process. To do this, we developed a dynamic database in order to store patient name, his/her kidney stage disease, age, adders, new sign and symptoms.

\section{The User Interface}

The user interface of the prototype system is allows a non-expert and physicians users to query (question) the KBS, and to see delivery of advices. In this prototype, users can interact with the system through "yes", "no", "what", "why" and, "how" responses. Based on the user response, the system draws a conclusion and displayed in the user interface window.

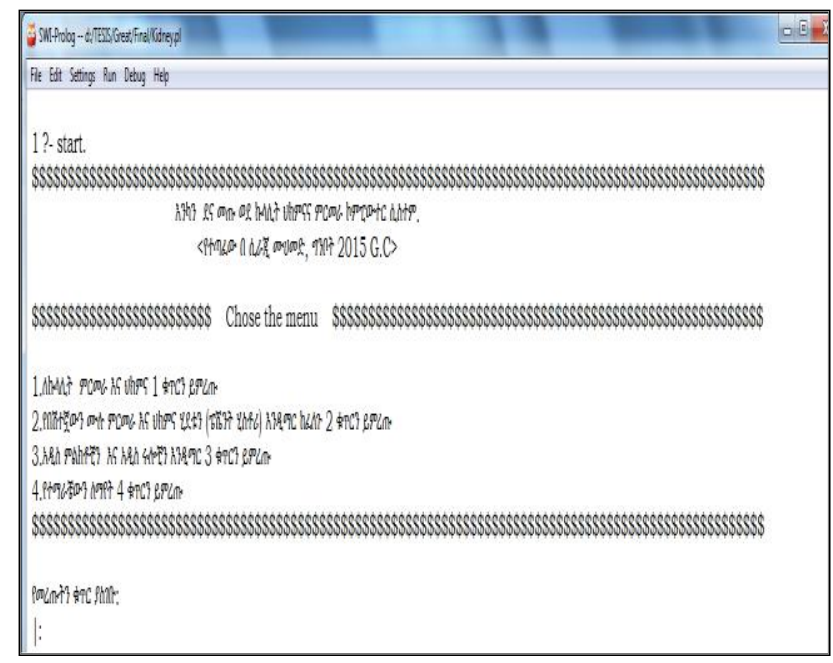

Fig.4. Welcoming Window for Prototype System

\section{Testing and Evaluations}

Developing a knowledge-based system is an incremental process. Therefore, in developing the prototype system, the knowledge engineer at finishing step should test the performance of the system. In this section, we discussed the testing and evaluation of the prototype system. In our research, we used test cases and visual interactions approaches. The results of this evaluation and test show where the system is weak. Also, the correct and incorrect outcomes are identified by comparing decisions made by domain experts with decisions made by the prototype system.

\section{A. Test Cases}

The test cases are used to measure the accuracy of the system. To measure the accuracy of the system, the researcher was selected 60 test cases as a representative of the domain. We categorized those cases into three stages based on the GFR laboratory result values and their symptoms. Naturally, evaluation of the knowledgebased system using test case needs experts as evaluators.

The knowledge-based system testing procedure carried out by system evaluator to classify the test cases into correct or incorrect classes. The evaluation was done by comparing the system test result with the physician answers (as the human expert did). Therefore, System evaluators and knowledge engineer made decisions by comparing the system test result with the physician answers. The result of the comparison shows that our 
approach has made a close decision as the human expert did. The following table 1 , shows the amount of test case and their exact class classifier.

Table 1. Total Taste Case with Accuracy Values.

\begin{tabular}{|l|l|l|l|l|l|}
\hline No. & $\begin{array}{l}\text { Selected } \\
\text { Cases }\end{array}$ & $\begin{array}{l}\text { Total } \\
\text { number of } \\
\text { cases }\end{array}$ & $\begin{array}{l}\text { Correctly } \\
\text { classified }\end{array}$ & $\begin{array}{l}\text { Incorrectly } \\
\text { classified }\end{array}$ & $\begin{array}{l}\text { Accuracy } \\
\text { in \% }\end{array}$ \\
\hline 1 & Stage-1 & 20 & 20 & 0 & $100 \%$ \\
\hline 2 & Stage-2 & 20 & 16 & 4 & $80 \%$ \\
\hline 3 & Stage-3 & 20 & 19 & 1 & $95 \%$ \\
\hline
\end{tabular}

Table 1, shows that from total of 60 test cases, in the first row show that, out of 20 diagnosed patients test cases in Stage-1, 20 test cases are classified as correct and 0 as incorrect. On the second row shows that, the total 20 diagnosed patients' test cases, 16 diagnosed patients taste cases are classified as correct and 4 test cases as incorrect. Also, the third rows show, that of the total 20 diagnosed patients' test cases, 19 diagnosed patients' test cases are classified as correctly classified and 1 as incorrectly classified. In general, from 60 diagnosed patients test cases 55 diagnosed patients' test cases are classified correctly and 5 diagnosed patients cases are classified incorrectly. The test case result that provided by system evaluators showed that the prototype system are about $91 \%$ correct and $9 \%$ incorrect.

\section{B. User Acceptance Evaluation}

The goal of user acceptance testing is to assess if the system can support day-to-day business, user scenarios and to ensure the system is sufficient and correct for business usage [22]. Visual interaction evaluation method are used when the domain expert to directly interact with the system. The evaluators assess the accuracy of the prototype system by using the following standards, these are:

$\checkmark$ Simplicity of use and interact with the prototype system,

$\checkmark$ Attractiveness of the prototype system,

$\checkmark$ efficiency in time, the accuracy of the prototype system in reaching a decision to identify the stage of kidney diseases,

$\checkmark$ The ability of the prototype system in making the right conclusions and recommendations, the ability of the prototype system to remember the patient history, and

$\checkmark$ The importance of the prototype system in the domain area.

We organized both closed-ended and open-ended questionnaires concerning on acceptance, attractiveness, time efficiency, accuracy of the prototype system, opinions, suggestions and feedback of experts. After evaluation the system the evaluators just put (ticked) their respond based on the researcher assigned values in numbers for each scale as excellent $=5$, very $\operatorname{good}=4$, good $=3$, fair $=2$, and poor $=1$ for each closed-ended questions. We used the following formulas to get the average values of the first close-ended user acceptance testing questions.

$$
1 * \frac{0}{5}+2 * \frac{0}{5}+3 * \frac{0}{5}+4 * \frac{2}{5}+5 * \frac{3}{5}=4.6
$$


Table 2. Illustrate The Performance Evaluation Rating Through Visual Interaction.

\begin{tabular}{|c|c|c|c|c|c|c|c|c|}
\hline No. & Criteria of evaluation & 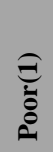 & 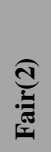 & 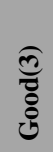 & 总 & 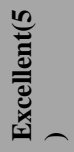 & 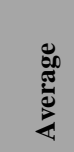 & 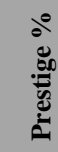 \\
\hline 1. & Is the prototype is easy to use and interact with it? & 0 & 0 & 0 & 2 & 3 & 4.6 & 92 \\
\hline 2. & How do you think the attractiveness of the prototype system? & 1 & 0 & 0 & 2 & 2 & 3.8 & 76 \\
\hline 3. & Is the system is more efficient in time? & 0 & 0 & 1 & 0 & 4 & 4.6 & 92 \\
\hline 4. & $\begin{array}{l}\text { How accuracy of the prototype system in reaching a decision to } \\
\text { identify the sages of kidney disease? }\end{array}$ & 0 & 0 & 1 & 2 & 2 & 4.2 & 84 \\
\hline 5. & Does the system incorporate sufficient and practical knowledge? & 0 & 0 & 0 & 3 & 2 & 4.4 & 88 \\
\hline 6. & Does the system making right conclusions and treatments? & 0 & 0 & 0 & 2 & 3 & 4.6 & 92 \\
\hline 7. & How do you rate the significance of the system in the domain area? & 0 & 0 & 0 & 1 & 4 & 4.8 & 96 \\
\hline 8. & $\begin{array}{l}\text { How do you rate the ability of a self-learning prototype system to } \\
\text { remember the patient's history? }\end{array}$ & 0 & 0 & 0 & 2 & 3 & 4.6 & 92 \\
\hline & & \multicolumn{5}{|c|}{ Total average } & 4.45 & 89 \\
\hline
\end{tabular}

As can be seen in table 2, for the simplicity and understandability of the information provided by the system, $40 \%$ of the respondents evaluated as a Very Good and $60 \%$ of them evaluated as an excellent system. For the attractiveness of the prototype system, $20 \%$ of them rated the system as poor, $40 \%$ of them ticked as the system very good and the remaining $40 \%$ rated as excellent. In the same way, for question "efficient in time" of the prototype system, $20 \%$ of them rated the system as good and $80 \%$ of them rated as excellent.

Similarly, for question "accuracy of the prototype system" of the prototype system, $20 \%$ of them rated the system as good, $40 \%$ of them ticked as the system very good and the remaining $40 \%$ rated as excellent. In the same sheet, the evaluators marked $60 \%$ as a very good system for "incorporate sufficient and practical knowledge", and $40 \%$ ticked as the system excellent. In the same way for criteria of the prototype system provide "making the right conclusions and treatments" $40 \%$ of them marked as very good and $60 \%$ of them marked as the system excellent. Similarly, for questions "rate the significance of the system in the domain area", $20 \%$ of the evaluator rated as very good and the remaining $80 \%$ of the evaluated marked as very good.

\section{Discussions and Recommendation}

The accuracy of the prototype system using test cases are calculated as $91 \%$ and the user acceptance testing using close-ended and open-ended questions average evaluation result is $89 \%$, respectively. Therefore, the overall accuracy performance of the prototype system is $90 \%$, because the system has Amharic language and self-learning features.

As stressed in the review of related work, some of the related works are conducted by Belay [23], Anteneh [24], Redeit [25], Solomon [26], Seblewongel [27], Zewditu [28], Guesh [12], and Solomon [17] in the area of medical in Ethiopia. Those local researchers recommend localizations concepts in terms of language and selflearning approaches. Therefore, conferring to their finding and suggestions this study has advancement, because the prototype system has Amharic language and self-learning ability to learn from experience which is enhance usability of the system. The recommendation section provides some clues to the interested researcher to investigate further approaches that are not covered in this research. Most of the time, a knowledge engineers have got some challenges when acquiring knowledge from experts, to solve that challenges the researcher suggested that for those who are interested to do research on the area knowledge acquisitions as future study, it is better using a self-learning KBS technology as knowledge acquisition approaches, because a self-learning KBS has the ability to hold all patient history. Correspondingly, this could be one approach for knowledge 
acquisition techniques to develop case-based reasoning applications. The prototype knowledge-based system has important modules for adding new rules, symptoms and learns from patient history but, rules that are found in knowledge base should be updated at run time without knowledge engineer involvement. Therefore, additional research needs to update the rules of the system automatically at knowledge base. The scope of this knowledge based system should be comprehensive to integrate others stages of kidney diseases, such as Stage4 and Stage-5 in order to be converted into a complete system. Furthermore, extract the necessary knowledge from experts was difficult for the researcher due to the personal nature of tacit knowledge. Therefore, it is important to apply data mining techniques to extract the hidden knowledge.

\section{Conclusion}

The knowledge engineer to develop a self-learning Amharic based KBS for diagnosis and treatments of kidney disease performed basic tasks: first the knowledge engineer assessed the problem area and the problem is considered feasible, then a statement of requirements is created and the development process continued as follows, knowledge was acquired using both structured and unstructured interviews with domain experts and from relevant documents by using documents analysis method to get concepts and facts of kidney diseases.

The acquired knowledge focuses on concepts and facts of kidney diseases. Then, those extracted facts and concepts were modeled by using decision tree approach and the model has been converted into productive rules and coded by using SWI-Prolog tool to form the knowledge-based system. Finally, the evaluating and testing result shows that, the overall performance of the prototype system registered $91 \%$ accurate result.

\section{References}

[1] Collins AJ, Li S, Gilbertson DT, et al. "Chronic kidney disease and cardiovascular disease in the Medicare population". Kidney Inter 2003.

[2] E.D. Kock. "Decentralizing the Codification of Rules in a Decision Support Expert Knowledge Base", MSc Thesis, University of Pretoria, Faculty of Engineering, Built Environment and Information Technology, South Africa, 2003.

[3] P-H. Speel, A. Th. Schreiber, W. van Joolingen, G.van Heijst, G.J.Beijer. "Conceptual Modeling for Knowledge-Based", Systems Unilever Research Vlaardingen, University of Amsterdam, CIBIT, Bolesian, 2013.

[4] Ting-Peng Liang. "Special section: Research in integrating learning capabilities into information system", Guest editor, USA, 2010.

[5] Solomom G. "A self-learning KBS for diagnosis and treatment of diabetes", MSc Thesis, Addis Ababa University School of Information Science, Ethiopia 2010.

[6] Ajith Abraham. "Rule-based Expert Systems", Oklahoma State University, Stillwater, OK, USA, 2010.

[7] R.A. Akerkar and P.S. Sajja. "Knowledge-Based Systems", Jones and Bartlett Publishers, USA, 2010.

[8] E.D. Kock. "Decentralizing the Codification of Rules in a Decision Support Expert Knowledge Base", MSc Thesis, University of Pretoria, Faculty of Engineering, Built Environment and Information Technology, South Africa, 2003.

[9] P.J. Tuso. "SERVE Athiopia", Available at: http://www.ncbi.nlm.nih.gov/pmc/articles/PMC2471814/\#bib10, Accessed date: June 05, 2011.

[10] Grmachew. "World Health Organization", WHO Humanitarian Appeal 2009 Country Profile, June 15, 2011.

[11] Mustafa Arici. "Clinical Assessment of a Patient with Chronic Kidney Disease Department of Nephrology”, Faculty of Medicine, Hacettepe University, Ankara 06100, Turkey, 2010. 
[12] P-H. Speel, A. Th. Schreiber, W. van Joolingen, G.van Heijst, G.J.Beijer. "Conceptual Modeling for Knowledge-Based", Systems Unilever Research Vlaardingen, University of Amsterdam, CIBIT, Bolesian.

[13] E.K.juuso and k.Leiviskä. "Adaptive Expert Systems for Metallurgical Processes". fac workshop on expert systems in mineral and metal processing, ESPOO, Finland, 26-28 august 1992.

[14] Anteneh W. "Prototype Knowledge Based System in Antiretroviral Therapy", MSc Thesis, Addis Ababa University School of Information Science, Ethiopia, 2004.

[15] Guesh, D. "Designing knowledge based system for blood transfusion", MSc Thesis, Addis Ababa University School of Information Science, Ethiopia, 2013.

[16] Seblewongel, E. "Prototype knowledge based system for anxiety mental disorder", MSc Thesis, Addis Ababa University School of Information Science, Ethiopia, 2012.

[17] Solomon. "Develop self-learning knowledge based system for diagnosis and treatment of diabetes", MSc Thesis, Addis Ababa University School of Information Science, Ethiopia, 2013.

[18] Mir Anamul Hasan, Khaja Md. Sher-E-Alam, Ahsan Raja Chowdhury. "Human Disease Diagnosis Using a Fuzzy Expert System”, Journal of Computing, Vol. 2, No. 6, June 2010, NY, USA, ISSN 21519617, 2010.

[19] Munirah, M., Yusof, R., Aziz,J., Chew, S. "Development of Online Children Skin Diseases Diagnosis System", 2013.

[20] Buchanan, g.b. and shortliffe, h.e. 1984. "Rule-based expert systems: the mycin experiments of the stanford heuristic programming project". In g.b. buchanan and h.e. shortliffe, eds., 2-67. 2010.

[21] B.F. Richard, G. Violetta, and O.L. Mieczyslaw. "On Features of Decision Trees as a Technique of Knowledge Modelling", Proceedings of the Workshop on Computer Science and Information Technologies CSIT ${ }^{e e} 99$, Moscow, Russia, 1999.

[22] Vince Bordo. "CBAP Overview of User Acceptance Testing (UAT) for Business Analysts (bas)", Gondar University College of Natural Science, Ethiopia, 2010.

[23] Belay. "Amharic based KBS for diagnosis and treatment of dental disease", MSc Thesis, Gondar University College of Natural Science, Ethiopia, 2006.

[24] Anteneh W. "Prototype Knowledge Based System in Antiretroviral Therapy". MSc Thesis, Addis Ababa University School of Information Science, Ethiopia, 2004.

[25] Redeit A. "Design and Development of a prototype Knowledge-Based System for HIV PreTest Counseling", MSc Thesis, Addis Ababa University School of Information Science, Ethiopia, 2006.

[26] Solomon D. "the Potential for Applying Knowledge-Based System for Diagnosis of Acute Respiratory Tract Infections". MSc Thesis, Addis Ababa University School of Information Science, Ethiopia, 2010.

[27] Seblewongel E. "Prototype Knowledge Based System for Anxiety Mental Disorder Diagnosis", MSc Thesis, Addis Ababa University School of Information Science, Ethiopia, 2011.

[28] Guesh D. "Designing a Knowledge-Based System for Blood Transfusion", MSc theisis, Addis Ababa University, School of Information Science, Ethiopia, 2012.

\section{Authors' Profile}

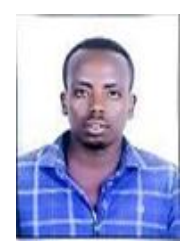

Siraj Mohammed Addis Ababa University, IT-Ph.D., Information retrieval Truck, Addis Ababa, Ethiopia.

Siraj Mohammed received the MS and BS degrees from the information technology Department, Gondar University, and Jimma University Ethiopia, in 2004 and 2006, respectively. $\mathrm{He}$ is a $\mathrm{PhD}$ candidate in the Department of information retrieval at Addis Ababa University of IT-PhD institute. His research interests include machine learning, semi supervised learning, and knowledge based system (expert system), information extraction and information retrieval and their applications. He is now working on his $\mathrm{PhD}$ research on information extraction. 
How to cite this paper: Siraj Mohammed,"A Self-learning Knowledge based System for Diagnosis and Treatment of Chronic Kidney Disease", International Journal of Education and Management Engineering(IJEME), Vol.9, No.2, pp.44-58, 2019.DOI: 10.5815/ijeme.2019.02.05 